\title{
1 Proteolytic Activation of Human-specific Olduvai Domains by the Furin
}

\section{Protease}

3 Ashley Pacheco ${ }^{1 \dagger}$, Aaron Issaian ${ }^{1 \dagger}$, Jonathan Davis ${ }^{1}$, Nathan Anderson ${ }^{1}$, Travis Nemkov ${ }^{1}$, Beat

$4 \quad$ Vögeli ${ }^{1}$, Kirk Hansen $^{1}$, James M. Sikela ${ }^{1 *}$

$5 \quad{ }^{1}$ Department of Biochemistry and Molecular Genetics, University of Colorado, Aurora, CO,

6 USA

7 These authors contributed equally to this work.

8

9 *Author for Correspondence: James M. Sikela, Department of Biochemistry and Molecular

10 Genetics, University of Colorado, Aurora, CO, USA, james.sikela@cuanschutz.edu

11 


\section{ABSTRACT}

13 Olduvai protein domains (formerly DUF1220) show the greatest human-specific increase in copy

14 number of any coding region in the genome and are highly correlated with human brain

15 evolution and cognitive disease. The majority of human copies are found within four NBPF

16 genes organized in a variable number of a tandemly arranged three-domain blocks called

17 Olduvai triplets. Here we show that these human-specific Olduvai domains are

18 posttranslationally processed by the furin protease, with a cleavage site occurring once at each

19 triplet. These findings suggest that all expanded human-specific $N B P F$ genes encode proproteins

20 consisting of many independent Olduvai triplet proteins which are activated by furin processing.

21 The exceptional correlation of Olduvai copy number and brain size taken together with our new

22 furin data, indicates the ultimate target of selection was a rapid increase in dosage of

23 autonomously functioning Olduvai triplet proteins, and that these proteins are the primary active

24 agent underlying Olduvai's role in human brain expansion. 


\section{Proteolytic Activation of Human-specific Olduvai Domains by the Furin}

\section{Protease}

\section{INTRODUCTION}

28 Olduvai protein domains (formerly called DUF1220) are approximately 65 amino acids in length

29 and are found almost exclusively within NBPF genes (Sikela and van Roy 2018). Studies have

30 shown that Olduvai copy number is linearly associated with primate brain size, neuron number,

31 and several other brain size-related phenotypes (Dumas and Sikela 2009; Dumas, et al. 2012;

32 Keeney, et al. 2015; Keeney, et al. 2014; Zimmer and Montgomery 2015). In humans, Olduvai

33 copy number is associated in a dosage-dependent manner to brain size, gray matter volume, and

34 cognitive aptitude (Davis, et al. 2015b; Dumas, et al. 2012). Olduvai domains have undergone

35 strong positive selection among primates and Olduvai copy number increases with evolutionary

36 proximity to humans (Popesco, et al. 2006). Non-primate mammals have 1-9 copies, monkeys

37 have 25-40 copies, great apes have 90-125 copies, and humans have 250-350 copies (O'Bleness,

38 et al. 2012). The hyper-amplification of Olduvai in humans represents the largest human-specific

39 increase in copy number of any coding region in the genome (O'Bleness, et al. 2012; Popesco, et

40 al. 2006; Zimmer and Montgomery 2015).

41 The majority of Olduvai domains follow a unique genomic organization that allows copy

42 number to be increased both rapidly and in an open-ended manner (Keeney, et al. 2014;

43 O'Bleness, et al. 2012). Olduvai domains are encoded by a small and large exon doublet (Finn, et

44 al. 2014; Popesco, et al. 2006; Vandepoele, et al. 2005) and can be phylogenetically subdivided

45 into six primary clades: conserved (CON1-3) and human lineage-specific (HLS1-3) (O'Bleness,

46 et al. 2012). The typical order of domain subtypes within human $N B P F$ genes is: one to several

47 CON1 domains; one CON2 domain; one-to-many instances of a triplet composed of HLS1, 
48 HLS2, and HLS3 domains; lastly one CON3 domain. The three-domain block of HLS sequences

49 is known as the Olduvai triplet (O'Bleness, et al. 2012; Sikela and Searles Quick 2018).

The rapid and extreme human lineage-specific Olduvai increase is predominantly the

51 result of tandem additions of Olduvai triplets within NBPF genes supplemented by gene

52 duplications after Olduvai triplet expansion (Fiddes, et al. 2019; O'Bleness, et al. 2012). The

53 proposed mechanism for the human expansion of $N B P F$ genes is through a recombination event

54 between HLS1 and CON3 which introduced a putative G-quadraplex (pG4) sequence pattern

55 (Heft, et al. 2020). G4 structures promote instability which in conjunction with selective pressure

56 is thought to have contributed to the human-specific extreme expansion of NBPF genes through

57 tandem additions of Olduvai triplets (Heft, et al. 2020). In NBPF genes that contain one or more

58 Olduvai triplets, the first triplet contains a canonical HLS1 small exon (HLS1-C), while every

59 additional triplet contains a larger HLS1 small exon (HLS1-E) that is also found in the CON3

60 subtype (Heft, et al. 2020). In addition to the pG4 sequence, the extended exon of HLS1 also

61 contains additional protein processing elements that have importance for human specific Olduvai

62 function and will be discussed in this work.

63 The Olduvai protein domain family has been proposed to represent a genomic trade-off

64 where selective pressure for increased copies resulted in increased brain size and cognition but,

65 in certain situations, copies can also vary in deleterious ways (Sikela and Searles Quick 2018).

66 Indeed, Olduvai copy number variation has been linked with multiple brain disorders including

67 autism spectrum disorder (ASD), macrocephaly, microcephaly and schizophrenia (Davis, et al.

68 2019; Davis, et al. 2015a; Davis, et al. 2014; Dumas and Sikela 2009; Dumas, et al. 2012;

69 Searles Quick, et al. 2015). Genomic data indicates that there are 23 human NBPF genes that

70 carry from 5 to 60 tandemly arranged Olduvai domains. Such an arrangement suggests that the 
71 proteins encoded by human NPBF genes would span a wide range of sizes. Surprisingly, this is

72 not the case. Rather Western analysis shows only a single primary band of $\sim 36 \mathrm{kDa}$. This result

73 has remained an enigma since it was first observed approximately 15 years ago (Popesco, et al.

74 2006). Here we present novel furin processing data confirming that expanded human-specific

$75 \quad N B P F$ genes are posttranslationally cleaved, converting larger NBPF proproteins into smaller

76 active protein units.

\section{RESULTS}

\section{Mass Spec Analysis and Validation of Olduvai HLS Peptide Size}

79 As mentioned above, predicted protein sizes of human NBPF proteins span a wide range, but

80 western blot analysis of human tissues shows only a single band for the HLS domains at $\sim 36 \mathrm{kDa}$

81 (Supplemental Figure 1A depicts previously published data from Popesco et al. 2006) (Popesco,

82 et al. 2006). This product could contain 3-4 Olduvai domains and suggests that the functional

83 size of HLS protein is smaller (Keeney, et al. 2014).

84 To further investigate this observation, the $36 \mathrm{kDa}$ band was enriched and analyzed by

85 mass spectrometry-based proteomics. Chromatographic pre-enrichment of the 36kDa band

86 combined with data-dependent global proteomic analysis identified a characteristic peptide of the

87 Olduvai HLS subtype (Supplemental Figure 1B). Subsequent targeted proteomics analysis was

88 able to identify additional peptides specific to NBPF including HLS domains (Supplemental

89 Figure 2). 


\section{Predicted Furin Processing Sites}

91 Analysis of potential protease cut sites determined that translated HLS1-E exons contain a

92 predicted furin cleavage site (Figure 1A). The HLS1-E exon, including the predicted furin site, is

93 found within every expanded Olduvai triplet and is absent from all unexpanded triplets (i.e. those

94 containing the HLS-C exon)(Figure 1B).

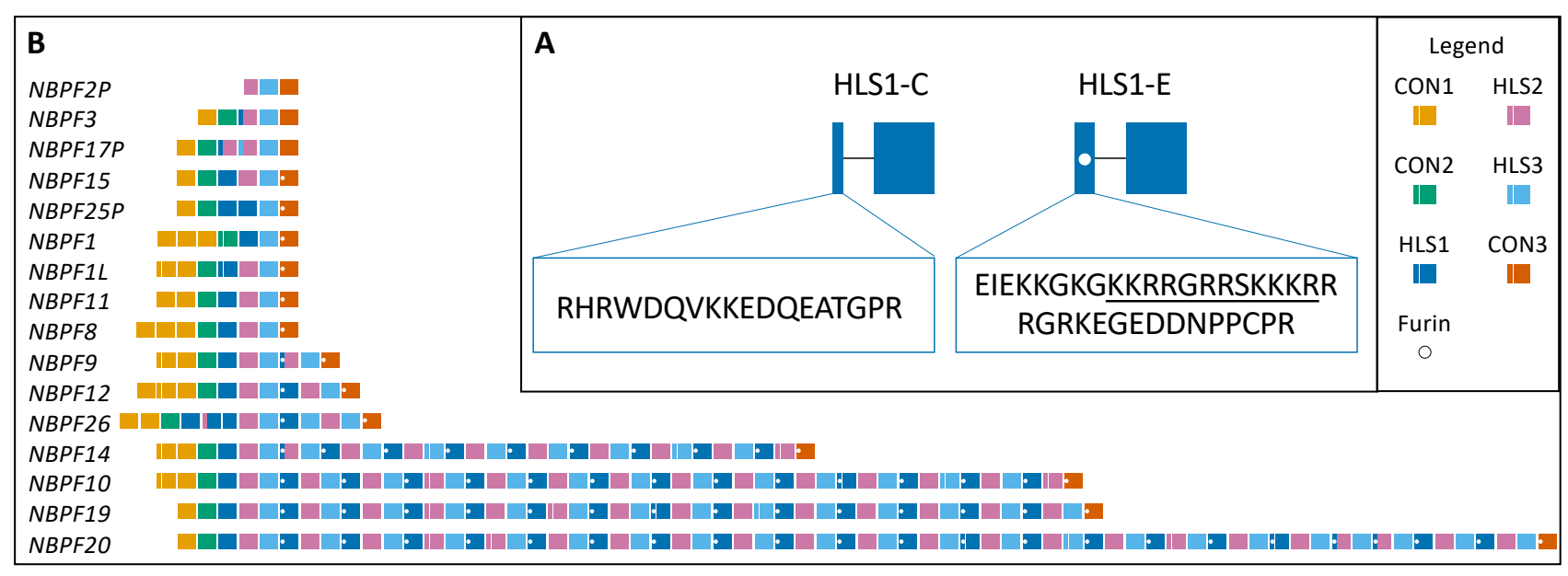

Fig. 1. (A) Analysis of protease cleavage sites predicted a furin site located in the small exon of HLS1-E indicated by underlined amino acid sequence and the white dot in the figure. The small exons of canonical HLS1 (HLS1-C) and expanded HLS1 (HLS1-E) domains are enlarged to show the translated amino acid sequence. (B) All expanded NBPF Olduvai triplets contain a predicted furin cleavage site located within the small exons of HLS1-E and CON3 indicated by the white dots in the figure. Figure adapted from Heft et al., 2020.

\section{Furin Protease Processing Cleaves Extended HLS Domains}

96 To evaluate potential furin processing of Olduvai triplets, both HLS1-C and HLS1-E were co-

97 expressed with furin. A single band is observed for HLS1-C while a multiple banding pattern is

98 observed for HLS1-E with furin co-expression (Figure 2A). These results confirm that extended

99 HLS1 domains are proteolytically processed by furin from longer 'proproteins'. The resulting 100 predicted protein products from furin processing would include the longer $\mathrm{N}$-terminal region of 101 the protein including CON1 and CON2 and Olduvai triplets with HLS1-C, all Olduvai triplets 
A

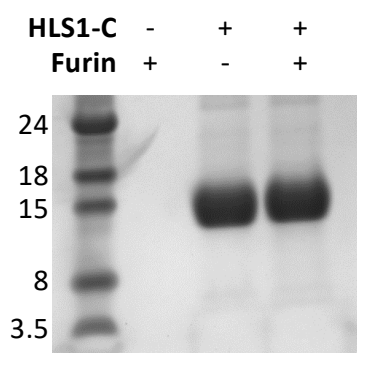

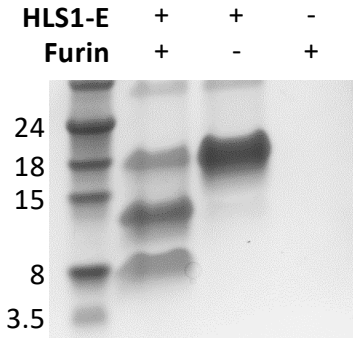

B

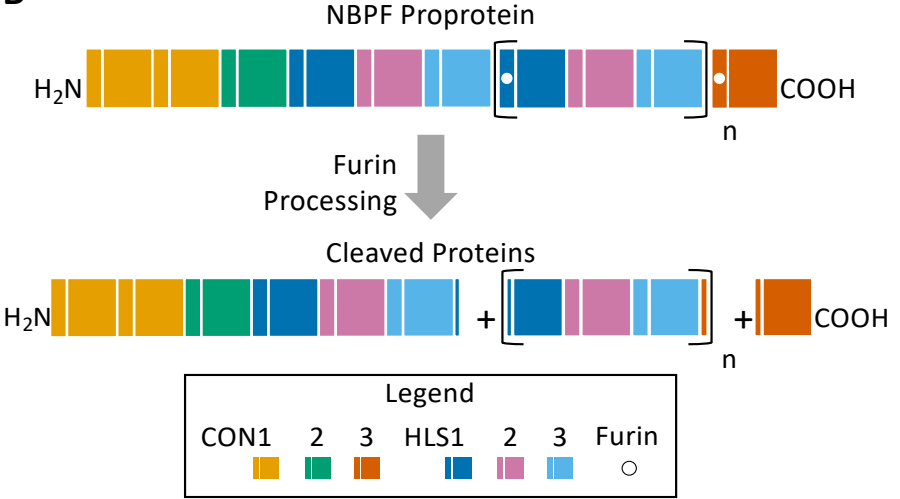

Fig. 2. (A) Furin is shown to cleave expanded HLS1 (HLS1-E). To analyze furin cleavage of HLS1, both HLS1-C and HLS1-E were independently co-expressed with furin. A single banding pattern is shown when HLS1-C is co-expressed with furin (as seen on the left), whereas a multiple banding pattern is seen when HLS1-E is co-expressed with furin (as seen on the right). This finding confirms the previously predicted furin cleavage site that is located within HLS1-E. (B) Furin processing of the NBPF proprotein would result in three categories of peptides: the NBPF N-terminal peptide, " $n$ " number of independent Olduvai triplet peptides, and the C-terminal CON3 peptide. A hypothetical NBPF protein with "n" number of Olduvai triplets is shown before and after furin processing. Furin cleavage sites are indicated by the white dots in HLS1-E and CON3. $\sim 28 \mathrm{kDa}$, which is lower than the observed $\sim 36 \mathrm{kDa}$ band. While a higher than expected observed molecular weight $(\mathrm{Mw})$ for this product could simply be due to structural peculiarities of the triplet protein, it may also indicate presence of posttranslational modifications (PTMs). It has

111 been previously determined that Olduvai domains are intrinsically disordered (Issaian, et al.

112 2019; Wu, et al. 2020). As protein phosphorylation is the most common form of PTM, and 
113 intrinsically disordered proteins are disproportionately phosphorylated relative to other proteins

114 (Bah and Forman-Kay 2016), predictive analysis for protein phosphorylation was conducted.

115 Prediction of protein phosphorylation sites shows the presence of multiple predicted sites within

116 the cleaved protein product, which could account for the increased observed Mw of the cleaved

117 Olduvai triplet protein (Supplemental Figure 3). This finding is preliminary and further

118 investigation will be needed to determine whether other forms of PTM are involved.

\section{DISCUSSION}

120 The evolutionary hyper-amplification of the Olduvai protein domain family in humans represents

121 one of the most dramatic human lineage-specific genomic events. Due to several recent studies,

122 we are beginning to understand the specific steps of how this occurred primarily through the

123 following two processes. The first is that human Olduvai expansion has employed a very rapid

124 and highly expandable mechanism for increasing copy number by addition of tandemly arrayed

125 Olduvai triplets (Heft, et al. 2020). The second being the addition of a furin cleavage site within

126 each triplet which allows expanded copies to be readily converted via posttranslational

127 processing to smaller independent active protein units, i.e., Olduvai triplets. It is likely that these

128 events were driven by strong evolutionary selection pressures as they are seen within several

129 human $N B P F$ genes, and have added over 50 Olduvai triplets to the human genome over the past

130 2-3 million years (Fiddes et al 2019). Taken together, these findings indicate that, rather than

131 simply raising copy number, the primary target of selection in humans has been to rapidly

132 increase the dosage of autonomously functioning Olduvai triplet proteins, and these are the

133 primary active agents of Olduvai function in humans.

134 Notably, processing by furin indicates that the N-terminal region including CON1 and

135 CON2 as well as the C-terminal CON3 may be separated from extended HLS domains after 
136 translation. This finding suggests that CON and HLS domains may have independent

137 functionality, which is supported by differing associations for various Olduvai domain subtypes.

138 For example, one of the most striking and well-supported findings is the linear association

139 between CON1 copy number and ASD severity (Davis, et al. 2019; Davis, et al. 2015a; Davis, et

140 al. 2014). This link is not found with any other Olduvai subtypes, despite their being adjacent to

$141 \mathrm{CON} 1$ in the NBPF proprotein. Independent function of the NBPF N-terminal protein product

142 may have relevance to associations between CON1 and ASD severity and is to be discussed in a

143 subsequent paper.

144 Finally, the new insights presented here resolve a long-standing question regarding the

145 function of Olduvai domains in humans, and show how selection has favored increasing levels of

146 individual Olduvai triplet proteins. How this dosage phenomenon is tied to the evolutionary

147 increase in human brain size is an interesting future area of investigation.

148 MATERIALS AND METHODS

149 Mass Spec Analysis

150 Untargeted proteomic identification was performed on protein digests using liquid

151 chromatography-tandem mass spectrometry as previously reported (Dzieciatkowska, et al. 2014).

152 Briefly, nanoflow reverse-phase LC-MS/MS was performed using an Eksigent nanoLC2D

153 system coupled to a LTQ Orbitrap-Velos mass spectrometer (Thermo Fisher). Data acquisition

154 was performed using XcaliburTM (Version 2.1, Thermo Fisher) software. Raw data files were

155 converted into Mascot generic files using PAVA. These peak lists were searched against

156 SwissProt human database using Mascot server (Version 2.2.06, Matrix Science). Mass

157 tolerances were $15 \mathrm{ppm}$ for MS peaks, and 0.5 Da for MS/MS fragment ions. 


\section{Enrichment of 36kDa Band}

159 To enrich the $36 \mathrm{kDa}$ protein with antibody positivity for proteomics analysis, $100 \mathrm{mg}$ of human

160 brain tissue (Keeney, et al. 2015) was extracted and subjected to a series of fractionations during

161 which the presence of the $36 \mathrm{kDa}$ band was followed by western analysis using an antibody

162 directed against the HLS subtype sequence. Antibody positive fractions were then dissolved in

$1630.1 \%$ formic acid and analyzed by targeted proteomics. Multiple reaction monitoring MRM was

164 performed using an LC-MS/MS system interfaced with a an UPLC system (QTRAP 5500 and

165 Ultimate 3000, respectively, Sciex and Thermo Fisher Scientific). The mass spectrometer was

166 run in positive ionization mode with the following settings: a source temperature of $200^{\circ} \mathrm{C}$, spray

167 voltage of $5300 \mathrm{~V}$, curtain gas of $20 \mathrm{psi}$, and a source gas of 35 psi (nitrogen gas). Multiple SRM

168 transitions were monitored using unit resolution in both Q1 and Q3 quadrupoles to maximize

169 specificity. SRM assay optimization was performed with the aid of computer software (Skyline,

170 Version 3.1). Collision energies and declustering potential were optimized for each transition.

171 Method building and acquisition were performed using the instrument-supplied software

172 (Analyst, Version 1.5.2, AB Sciex). Raw SRM data files were imported to Skyline Version 3.1

173 software for data processing.

\section{Protein Expression and Purification}

175 Codon optimized gene fragments of HLS-C and HLS-E were cloned into pET21b(+) with the

176 NdeI and XhoI restriction sites. Each expression construct contained an N-terminal MBP tag, a

177 TEV cleavage site, and C-terminal 6xHis-tag. The plasmids were transformed and expressed in

178 Escherichia coli strain Rosetta ${ }^{\mathrm{TM}}$ 2(DE3)pLysS cells with appropriate antibiotic selection. All

179 bacterial expressed recombinant proteins were grown in Luria-Bertani (LB) broth 
HLS-C and HLS-E were expressed as follows. Three colonies from an overnight culture plate were resuspended in $1.5 \mathrm{~L}$ of LB with $0.001 \%$ Antifoam 204 and shaken at $26{ }^{\circ} \mathrm{C}$ for 14 hours. The A600 was measured to ensure the culture was below 0.5. Fresh LB was inoculated with the overnight culture $(3: 1)$ and shaken at $37^{\circ} \mathrm{C}$. The incubator temperature was lowered to $25^{\circ} \mathrm{C}$ once the A600 reached 0.4. The culture was induced with $0.5 \mathrm{mM}$ Isopropyl $\beta$ - d-1thiogalactopyranoside (IPTG) once the A600 reached 0.6 and shaken for 6 hours at $25^{\circ} \mathrm{C}$. Cells were harvested by centrifugation at $4{ }^{\circ} \mathrm{C}$ for $10 \mathrm{~min}$ at $4500 \mathrm{rpm}$.

187 HLS-C and HLS-E were purified as follows. Cell pellets were resuspended in $50 \mathrm{mM}$ Tris (pH 7.5), $300 \mathrm{mM} \mathrm{NaCl}, 8 \mathrm{M}$ urea, $2 \%$ glycerol, $10 \mathrm{mM}$ imidazole, and $10 \mathrm{mM} \beta \mathrm{ME}$. Cell disruption was performed by sonication $(7 \times 20 \mathrm{~s})$ at $4{ }^{\circ} \mathrm{C}$. The lysates were clarified by centrifugation at 13,000 rpm for $30 \mathrm{~min}$ at $4{ }^{\circ} \mathrm{C}$ and loaded onto a pre-equilibrated column packed with $\mathrm{Ni}$

191 Sepharose ${ }^{\mathrm{TM}}$ excel resin. The column was washed with $5 \mathrm{CV}$ of resuspension buffer and His192 tagged proteins were eluted with $50 \mathrm{mM}$ Tris ( $\mathrm{pH} 7.5), 50 \mathrm{mM} \mathrm{NaCl}, 8 \mathrm{M}$ urea, $3 \%$ glycerol, 193 and $400 \mathrm{mM}$ imidazole. The protein sample was diluted and loaded onto a pre-equilibrated 194 column packed with Source ${ }^{\mathrm{TM}}$ 15Q. Proteins were separated using the following gradient: $0-50$ $\% \mathrm{~B}(0-12 \mathrm{CV}), 50-75 \% \mathrm{~B}(12-15 \mathrm{CV}), 75-100 \% \mathrm{~B}(15-15.5 \mathrm{CV}), 100 \% \mathrm{~B}(15.5-$

$19616.5 \mathrm{CV})$ with buffer A (20 mM Tris (pH 8.0), $8 \mathrm{M}$ urea) and buffer B (20 mM Tris (pH 8.0), 8

$197 \mathrm{M}$ urea, $1 \mathrm{M} \mathrm{NaCl})$. The relevant fractions were pooled and dialyzed overnight at $4{ }^{\circ} \mathrm{C}$ against 20 $198 \mathrm{mM}$ Tris (pH 7.5), $50 \mathrm{mM} \mathrm{NaCl}$, and $5 \mathrm{mM}$ DTT. TEV protease (in-house) was added to the 199 sample and incubated overnight at $4{ }^{\circ} \mathrm{C}$ with gentle rocking. The protein sample was loaded onto 200 a pre-equilibrated column packed with Source ${ }^{\mathrm{TM}}$ 15Q. Proteins were separated using the 201 following gradient: $0-50 \% \mathrm{~B}(0-12 \mathrm{CV}), 50-75 \% \mathrm{~B}(12-15 \mathrm{CV}), 75-100 \% \mathrm{~B}(15-15.5$ $202 \mathrm{CV}), 100 \% \mathrm{~B}(15.5-16.5 \mathrm{CV})$ with buffer A (20 mM Tris (pH 8.0), $8 \mathrm{M}$ urea) and buffer B (20 
$\mathrm{mM}$ Tris ( $\mathrm{pH} 8.0$ ), $8 \mathrm{M}$ urea, $1 \mathrm{M} \mathrm{NaCl}$ ). Relevant fractions were pooled and dialyzed overnight at $4{ }^{\circ} \mathrm{C}$ against $20 \mathrm{mM}$ HEPES (pH 7.5), $200 \mathrm{mM} \mathrm{NaCl}$, and $5 \mathrm{mM}$ DTT. The samples were concentrated to $1.5 \mathrm{~mL}$ using a $3000 \mathrm{MWCO}$ concentrator (Sartorius) and diluted to $3 \mathrm{~mL}$ with urea buffer (20 mM Tris (pH 7.5), $300 \mathrm{mM} \mathrm{NaCl,} 8 \mathrm{M}$ urea, $5 \mathrm{mM}$ DTT). The samples were

207 injected onto a Superose ${ }^{\mathrm{TM}} 6$ Increase column (Cytiva) pre-equilibrated with native storage

208 buffer (20 mM HEPES (pH 7.5), $200 \mathrm{mM} \mathrm{NaCl,} 5 \mathrm{mM}$ DTT). Fractions containing pure target

209 protein were pooled, concentrated, and stored at $-80{ }^{\circ} \mathrm{C}$.

210 In-Solution Enzymatic Digestion

211 Both HLS-C and HLS-E protein at $1 \mathrm{mg} / \mathrm{mL}$ were incubated with human Furin (EC 3.4.21.75,

212 P09958, obtained from NEB P8077) in digestion buffer (10 mM HEPES (pH 7.5), $0.2 \mathrm{mM}$

$213 \mathrm{CaCl} 2,0.2 \mathrm{mM} \beta \mathrm{ME}, 0.1 \%$ Triton $\mathrm{X}-100)$ for 16 hours at $\mathrm{RT}\left(25^{\circ} \mathrm{C}\right)$. Additional samples

214 lacking either Olduvai Triplet or Furin were assembled as experimental controls. Samples were

215 mixed with 1X LDS Sample Buffer (GenScript) supplemented with $100 \mathrm{mM} \beta \mathrm{ME}$ and incubated

216 at $95{ }^{\circ} \mathrm{C}$ for $5 \mathrm{~min}$. Equal volumes of each sample were loaded onto a 4-12\% SDS-PAGE gel

217 along with the BLUEstainTM 2 (GoldBio) protein ladder. The gel was stained with

218 SimplyBlueTM Safe Stain (Thermo Fisher Scientific) and visualized with a CCD equipped

219 imager (Bio-Rad).

220 In-situ Analyses

221 Furin site prediction was carried out by submitting full length NBPF protein sequences to the

222 ProP - 1.0 server (Duckert, et al. 2004). Protein phosphorylation site prediction was conducted

223 by submitting Olduvai triplet protein sequences containing cleaved HLS1-E exons to the

224 NetPhos - 3.1 server(Blom, et al. 1999). 


\section{ACKNOWLEDGEMENTS}

226 We thank Ilea Heft, Morkos Henen and Natasia Paukovich for helpful discussions and Monika

227 Dzieciatkowska for assistance with mass spectrometry experiments. This work was supported by

228 the National Institute of General Medical Sciences at the National Institutes of Health

229 (R01GM130694-01A1 to B.V.), and the National Institute of Mental Health at the National

230 Institute of Health (R01MH108684 to J.M.S.).

231

232 COMPETING INTERESTS

233 The authors declare that they have no conflict of interest.

\section{DATA AVAILABILITY STATEMENT}

236 The data underlying this article are available in the article and in its online supplementary

237 material. 


\section{References}

239 Bah A, Forman-Kay JD 2016. Modulation of Intrinsically Disordered Protein Function by Post-

240 translational Modifications. J Biol Chem 291: 6696-6705. doi: 10.1074/jbc.R115.695056

241 Blom N, Gammeltoft S, Brunak S 1999. Sequence and structure-based prediction of eukaryotic

242 protein phosphorylation sites. J Mol Biol 294: 1351-1362. doi: 10.1006/jmbi.1999.3310

243 Davis JM, Heft I, Scherer SW, Sikela JM 2019. A Third Linear Association Between Olduvai

244 (DUF1220) Copy Number and Severity of the Classic Symptoms of Inherited Autism. Am J

245 Psychiatry 176: 643-650. doi: 10.1176/appi.ajp.2018.18080993

246 Davis JM, Searles Quick VB, Sikela JM 2015a. Replicated linear association between DUF1220

247 copy number and severity of social impairment in autism. Hum Genet 134: 569-575. doi:

$248 \quad 10.1007 / \mathrm{s} 00439-015-1537-6$

249 Davis JM, et al. 2014. DUF1220 dosage is linearly associated with increasing severity of the

250 three primary symptoms of autism. PLoS Genet 10: e1004241. doi:

251 10.1371/journal.pgen.1004241

252 Davis JM, et al. 2015b. DUF1220 copy number is linearly associated with increased cognitive

253 function as measured by total IQ and mathematical aptitude scores. Hum Genet 134: 67-75. doi:

254 10.1007/s00439-014-1489-2

255 Duckert P, Brunak S, Blom N 2004. Prediction of proprotein convertase cleavage sites. Protein

256 Eng Des Sel 17: 107-112. doi: 10.1093/protein/gzh013

257 Dumas L, Sikela JM 2009. DUF1220 domains, cognitive disease, and human brain evolution.

258 Cold Spring Harb Symp Quant Biol 74: 375-382. doi: 10.1101/sqb.2009.74.025

259 Dumas LJ, et al. 2012. DUF1220-domain copy number implicated in human brain-size

260 pathology and evolution. Am J Hum Genet 91: 444-454. doi: 10.1016/j.ajhg.2012.07.016 
261 Dzieciatkowska M, Hill R, Hansen KC. 2014. GeLC-MS/MS Analysis of Complex Protein

262 Mixtures. In: Martins-de-Souza D, editor. Shotgun Proteomics: Methods and Protocols. New

263 York, NY: Springer New York. p. 53-66.

264 Fiddes IT, Pollen AA, Davis JM, Sikela JM 2019. Paired involvement of human-specific

265 Olduvai domains and NOTCH2NL genes in human brain evolution. Hum Genet 138: 715-721.

266 doi: 10.1007/s00439-019-02018-4

267 Finn RD, et al. 2014. Pfam: the protein families database. Nucleic Acids Res 42: D222-230. doi:

268 10.1093/nar/gkt1223

269 Heft IE, et al. 2020. The Driver of Extreme Human-Specific Olduvai Repeat Expansion Remains

270 Highly Active in the Human Genome. Genetics 214: 179-191. doi: 10.1534/genetics.119.302782

271 Issaian A, et al. 2019. Solution NMR backbone assignment reveals interaction-free tumbling of

272 human lineage-specific Olduvai protein domains. Biomol NMR Assign 13: 339-343. doi:

273 10.1007/s12104-019-09902-0

274 Keeney JG, et al. 2015. DUF1220 protein domains drive proliferation in human neural stem cells

275 and are associated with increased cortical volume in anthropoid primates. Brain Struct Funct

276 220: 3053-3060. doi: 10.1007/s00429-014-0814-9

277 Keeney JG, Dumas L, Sikela JM 2014. The case for DUF1220 domain dosage as a primary

278 contributor to anthropoid brain expansion. Front Hum Neurosci 8: 427. doi:

$279 \quad 10.3389 /$ fnhum.2014.00427

280 O'Bleness MS, et al. 2012. Evolutionary history and genome organization of DUF1220 protein

281 domains. G3 (Bethesda) 2: 977-986. doi: 10.1534/g3.112.003061

282 Popesco MC, et al. 2006. Human lineage-specific amplification, selection, and neuronal

283 expression of DUF1220 domains. Science 313: 1304-1307. doi: 10.1126/science.1127980 
284 Searles Quick VB, Davis JM, Olincy A, Sikela JM 2015. DUF1220 copy number is associated

285 with schizophrenia risk and severity: implications for understanding autism and schizophrenia as

286 related diseases. Transl Psychiatry 5: e697. doi: 10.1038/tp.2015.192

287 Sikela J, van Roy F 2018. Changing the name of the NBPF/DUF1220 domain to the Olduvai

288 domain [version 2; peer review: 3 approved]. F1000Research 6. doi:

289 10.12688/f1000research.13586.2

290 Sikela JM, Searles Quick VB 2018. Genomic trade-offs: are autism and schizophrenia the steep

291 price of the human brain? Hum Genet 137: 1-13. doi: 10.1007/s00439-017-1865-9

292 Vandepoele K, Van Roy N, Staes K, Speleman F, van Roy F 2005. A novel gene family NBPF:

293 intricate structure generated by gene duplications during primate evolution. Mol Biol Evol 22:

294 2265-2274. doi: 10.1093/molbev/msi222

295 Wu H, Zhai LT, Guo XX, Rety S, Xi XG 2020. The N-terminal of NBPF15 causes multiple

296 types of aggregates and mediates phase transition. Biochem J 477: 445-458. doi:

297 10.1042/BCJ20190566

298 Zimmer F, Montgomery SH 2015. Phylogenetic Analysis Supports a Link between DUF1220

299 Domain Number and Primate Brain Expansion. Genome Biol Evol 7: 2083-2088. doi:

$300 \quad 10.1093 /$ gbe/evv122

301 